\title{
Conceptos básicos sobre el estudio de paternidad
}

\author{
MARCELA LAGOS L. ${ }^{1}$, HELENA POGGI M. ${ }^{1, a}$, \\ CECILIA MELLADO S. ${ }^{2}$
}

${ }^{1}$ Departamento de Laboratorios Clínicos.

${ }^{2}$ Departamento de Pediatría.

Facultad de Medicina, Pontificia Universidad Católica de Chile. Santiago de Chile. aioquímica.

Recibido el 24 de marzo de 2011, aceptado el 30 de marzo de 2011

Correspondencia: Dra. Marcela Lagos L. Laboratorio de Biología Molecular. Departamento de Laboratorios Clínicos Facultad de Medicina. Pontificia Universidad Católica de Chile. Vicuña Mackenna 4686, Macul. Santiago, Chile. Tel.: 3548515 - 3548584 Fax: 5523464 - 3548603 E-mail:mlagos@med. puc.cl

\section{Basic concepts about paternity testing}

Nowadays, the analysis of genetic markers is a very important and validated tool for the identification of individuals, and for paternity testing. To do so, highly variable regions of the human genome are analyzed, making it possible to obtain the genetic profile of an individual, and to distinguish between different individuals. The methodology used is basically the same all over the world, consisting in the analysis of 13 to 15 markers. To assign biological paternity the child must have inherited the characteristics from the alleged father in each of the genetic markers analyzed. This analysis achieves a certainty higher than with any other test, which is expressed as the probability of paternity. This probability has to be at least 99.9\%, but greater probabilities are usually obtained, especially if the mother is included in the analysis. If the characteristics of two or more genetic markers from the alleged father are absent in the child, biological paternity is excluded.

(Rev Med Chile 2011; 139: 542-547).

Key words: Forensic genetics; Genetic testing; Paternity.

\section{RESUMEN}

El análisis de marcadores genéticos se ha convertido en una herramienta muy importante y ampliamente reconocida para la identificación de individuos y para el estudio de paternidad. Para esto se estudian distintas regiones del genoma humano que son altamente variables en la población y que permiten obtener el perfil genético y distinguir entre distintos individuos. La metodología que se utiliza es básicamente la misma en todo el mundo y consiste en el análisis de entre 13 a 15 marcadores. La paternidad biológica se asigna cuando el hijo/a presenta las características que debe heredar del presunto padre en cada uno de los marcadores genéticos estudiados. A través de este análisis es posible asignar paternidad con un grado de certeza más alto que con cualquier otro sistema, el que se expresa como probabilidad de paternidad. Esta probabilidad debe alcanzar al menos 99,9\%. Sin embargo, es posible obtener probabilidades mucho más altas, sobre todo si se incluye a la madre en el estudio. Si las características genéticas del supuesto padre están ausentes en el hijo/a en al menos dos marcadores, se excluye la paternidad biológica. 


\section{DE LA VIDA REAL}

Un hombre de 30 años, soltero, tiene dudas sobre la paternidad de un menor de 3 años y se realiza estudio de paternidad. En el informe de resultados se indican las fechas de toma de muestra, los datos personales, los distintos marcadores genéticos estudiados y los alelos presentes en el hijo y en el padre. En la conclusión del estudio se indica que la probabilidad de paternidad es de 99,89\%. El estudio no incluye a la madre, quien no quiso tomarse muestra de sangre, pero autorizó el estudio del niño, ya que no está reconocido por el padre. Madre y padre le consultan por la interpretación de estos resultados y el grado de certeza del examen.

¿Con qué grado de certeza se puede asignar paternidad y cómo se expresa?

¿Es importante incluir a la madre en un estudio de paternidad?

\section{Un problema antiguo con soluciones nuevas}

Desde los tiempos del Imperio Romano hay registros sobre disputas por la paternidad, con fines del pago de alimentos o de herencia, pero también con el sólo fin de que ésta sea reconocida. Sin embargo, existían dificultades importantes para probar la paternidad, ya que "las pruebas" se basaban principalmente en el parecido físico. $\mathrm{La}$ posibilidad de asignar o excluir paternidad era tan incierta que, por ejemplo, en Francia a comienzos del siglo XIX se prohibieron las demandas por paternidad, basándose en el principio de pater semper incertus ${ }^{1}$. En la primera mitad del siglo XX hubo avances importantes, dado que fue posible contar con herramientas objetivas como la determinación de los grupos sanguíneos que permitían excluir paternidad claramente, pero asignar paternidad con altos niveles de certeza aun seguía siendo un desafío. Recién en los últimos 20 años se produjeron los avances tecnológicos que han tenido el mayor impacto en el estudio de paternidad gracias a la identificación de individuos basada en el análisis de determinadas regiones del ADN.

\section{El perfil genético}

Como organismos diploides que somos, todos los seres humanos poseemos dos sets de información genética, uno heredado del padre y otro de la madre, por lo tanto, la información genética del padre biológico debe estar presente en el hijo. Esta información está contenida en los cromosomas y su ADN, y tiene una alta homología entre indivi- duos (99,9\%). Sin embargo, hay regiones $(0,1 \%)$ que son altamente variables y que nos diferencian de otros individuos, sobre cuya base es posible obtener el perfil genético de un individuo ${ }^{2}$. A la variante heredada de uno o el otro progenitor se le denomina alelo materno o paterno.

La identificación de individuos basada en el estudio de ADN se puede realizar a partir de prácticamente cualquier muestra biológica: sangre, mucosa bucal, pelo, orina, dientes o incluso de material biológico degradado. Es por eso que este análisis no sólo es útil para el estudio de paternidad/maternidad y otros parentescos ${ }^{3}$, sino que también se utiliza en estudios forenses, en investigaciones históricas y estudios antropológicos, así como también se aplica en otras áreas de la medicina como el análisis de quimerismo post-trasplante de médula ósea ${ }^{4}$.

\section{¿Qué regiones del genoma se estudian y cómo?}

Las regiones variables o polimórficas que se utilizan actualmente para identificación humana son secuencias cortas repetidas en tándem o STR (Short Tandem Repeats). Las secuencias utilizadas se componen de unidades que contienen cada una 4 a 5 pares de bases nucleotídicas (por ejemplo, ACAG) y lo que varía es el número de veces que estas unidades se repiten en cada individuo ${ }^{5}$. En el ejemplo de la Figura 1, el hijo hereda de la madre un alelo con 15 repeticiones, lo que implica que del padre recibió el alelo con 18 repeticiones; a este alelo que necesariamente se hereda del padre dado que se conoce el alelo de la madre, se le llama 


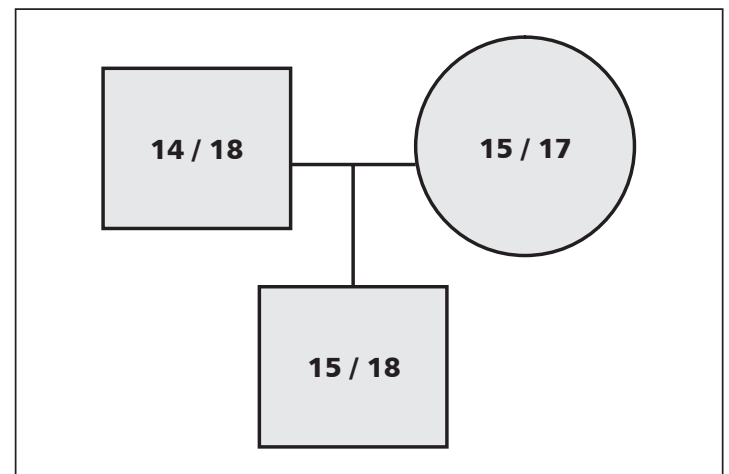

Figura 1. Ejemplo de un caso de paternidad (padre-madrehijo) con una distribución de los alelos en un STR que no permite excluir paternidad.

alelo paterno obligado. Aunque estas regiones son polimórficas, el número de repeticiones de un determinado STR no es único de un individuo, es decir, el alelo paterno obligado del hijo/a puede co- incidir por azar con uno de los alelos del supuesto padre, por lo que es necesario estudiar varios STR conjuntamente.

La metodología que se utiliza es básicamente la misma en todo el mundo y consiste en el análisis de entre 13 a 15 STR distribuidos en su mayoría en distintos cromosomas, así como un STR adicional para identificar el sexo ${ }^{6}$ (Tabla 1). Todas estas regiones se analizan simultáneamente, utilizando una metodología llamada reacción en cadena de la polimerasa o PCR (Polymerase Chain Reaction), con la que se obtienen millones de copias de cada región. Esto permite el análisis de cantidades muy pequeñas de $\mathrm{ADN}$, lo que es especialmente importante en las investigaciones forenses. Los productos de la PCR, que varían en el tamaño de acuerdo al número de repeticiones que cada individuo tenga, se separan por electroforesis capilar y se detectan por fluorescencia. Finalmente, se obtiene el número de repeticiones que presentan el presunto padre, la madre y el hijo/a para cada uno de los STR estudiados.

Tabla 1. STR habitualmente utilizados para el estudio de paternidada

\begin{tabular}{|lccc|}
\hline Nombre de la región & Cromosoma & Rango de $\mathbf{n}$ de repeticiones & n de alelos observados \\
\hline TPOX & 2 & $6-13$ & 10 \\
\hline D2S1338* & 2 & $11-28$ & 22 \\
\hline D3S1358 & 3 & $9-20$ & 20 \\
\hline FGA & 4 & $15-51,2$ & 69 \\
\hline CSF1PO & 5 & $6-16$ & 15 \\
\hline D5S818 & 5 & $7-16$ & 10 \\
\hline D7S820 & 7 & $6-15$ & 22 \\
\hline D8S1179 & 8 & $8-19$ & 13 \\
\hline TH01 & 11 & $3-14$ & 20 \\
\hline VWA & 12 & $10-24$ & 28 \\
\hline D13S317 & 13 & $5-15$ & 14 \\
\hline Penta E** & 15 & $5-26$ & 39 \\
\hline D16S539 & 16 & $5-15$ & 10 \\
\hline D18S51 & 18 & $7-27$ & 43 \\
\hline D19S433* & 19 & $5,2-20$ & 30 \\
\hline D21S11 & 21 & $24-38$ & 70 \\
\hline Penta D** & 21 & $1,1-18$ & 39 \\
\hline
\end{tabular}

aLos STR reciben el nombre de acuerdo a su ubicación. Por ejemplo: VWA por el factor von Willebrand o D3S1358 ya que corresponde a una determinada región en el cromosoma $3 .{ }^{*}$ incluidos en ensayo comercial AmpFISTR ${ }^{\circledR}$ Identifiler $^{\top M}$ (Applied Biosystems). **incluidos en ensayo comercial Powerplex ${ }^{\circledR} 16$ (Promega). 


\section{¿Con qué certeza se excluye o asigna paternidad?}

Una vez que se han comparado los perfiles del hijo/a con los del supuesto padre y hay dos o más STR en que no se observan alelos que tengan el mismo número de repeticiones, se excluye la paternidad y no se requiere realizar ningún cálculo probabilístico. En Chile, al igual que en otros países, se exige que la exclusión de paternidad se demuestre en al menos dos marcadores ${ }^{7}$, aunque por lo general, es posible excluir la paternidad en base a más marcadores. En el ejemplo de la Tabla 2 , que no incluye a la madre, hay 6 STR en que el hijo/a no presenta alelos del supuesto padre, lo que excluye la paternidad. En los otros STR, los supuestos padre e hijo/a comparten alelos con el mismo número de repeticiones por azar.

El hecho que no se excluya paternidad en base a sólo un marcador, se debe a que existe la posibilidad de que, de una generación a otra, ocurran cambios o mutaciones en el ADN que afecten los resultados en ese marcador. Como consecuencia,

Tabla 2. Perfiles genéticos de un supuesto padre y su hijo/a en un caso de exclusión de paternidad utilizando 15 STR

\begin{tabular}{|lcccc|}
\hline Marcadores & \multicolumn{2}{c}{$\begin{array}{c}\text { Alelos del } \\
\text { padre }\end{array}$} & \multicolumn{2}{c|}{$\begin{array}{c}\text { Alelos del } \\
\text { hijo }\end{array}$} \\
\hline D3S1358 & 14 & 15 & 15 & 15 \\
VWA & 16 & 16 & 16 & 18 \\
\hline FGA & $\mathbf{2 3}$ & $\mathbf{2 7}$ & $\mathbf{2 1}$ & $\mathbf{2 2}$ \\
\hline D8S1179 & 10 & 13 & 10 & 11 \\
\hline D21S11 & $\mathbf{3 2 , 2}$ & $\mathbf{3 3 , 2}$ & $\mathbf{2 9}$ & $\mathbf{3 1}$ \\
\hline D18S51 & $\mathbf{1 5}$ & $\mathbf{2 1}$ & $\mathbf{1 7}$ & $\mathbf{1 8}$ \\
\hline D5S818 & 9 & 10 & 10 & 11 \\
\hline D13S317 & $\mathbf{8}$ & $\mathbf{1 4}$ & $\mathbf{1 0}$ & $\mathbf{1 1}$ \\
\hline D7S820 & 10 & 10 & 10 & 11 \\
\hline D16S539 & 11 & 13 & 11 & 12 \\
\hline TH01 & $\mathbf{7}$ & $\mathbf{8}$ & $\mathbf{6}$ & $\mathbf{9}$ \\
\hline TPOX & 8 & 8 & 8 & 8 \\
\hline CSF1PO & $\mathbf{1 1}$ & $\mathbf{1 1}$ & $\mathbf{1 0}$ & $\mathbf{1 2}$ \\
\hline D2S1338 & 21 & 25 & 19 & 25 \\
\hline D19S433 & 14 & 14 & 13 & 14 \\
\hline
\end{tabular}

En negrita: marcadores y los alelos que permiten excluir la paternidad. es posible que el padre presente un alelo que no se observe en el hijo, aun siendo el padre biológico. Estas mutaciones son poco frecuentes y los laboratorios que realizan este examen tienen como norma confirmar los resultados con un segundo análisis cuando esto sucede.

En el ejemplo de la Tabla 3, el hijo/a presenta en todos los STR un alelo heredado de la madre y el otro del presunto padre, por lo que se asigna paternidad. La certeza, sin embargo, no es de $100 \%$, dado que teóricamente no se puede excluir la posibilidad que otro sujeto en la población posea el mismo perfil genético para los STR estudiados.

Para estimar el grado de certeza con que se asigna la paternidad es necesario contrastar la probabilidad que los alelos presentes en el hijo provengan del presunto padre versus la probabilidad que los haya recibido de cualquier otro individuo en la población. De acuerdo a eso, se calcula la razón entre ambas probabilidades, denominada índice de paternidad (IP), que expresa cuantas veces es más probable que el presunto padre sea el padre biológico a que lo sea cualquier otro sujeto en la población. El IP se modificará principalmente en función de la frecuencia con que se observen los alelos heredados del padre en la población general; a menor frecuencia alélica, mayor el IP y mayor la probabilidad de que sea el padre biológico. En el ejemplo de la Tabla 3, el IP en el STR D8S1179 es de 4,26 , es decir, es 4,26 veces más probable que el supuesto padre sea el padre biológico respecto a que lo sea otro sujeto de la población elegido al azar. En cambio, para el STR TPOX el IP alcanza sólo 1,06 ya que en este caso el alelo heredado del padre es más frecuente en la población.

Dado que los STR incluidos en un estudio de paternidad se heredan en forma independiente, es posible calcular la probabilidad para todos los STR en conjunto, multiplicando los IP obtenidos para cada STR (IP combinado). A partir del IP combinado, se calcula la probabilidad de paternidad (PP) según la fórmula: $\mathrm{PP}=\mathrm{IP} / \mathrm{IP}+1$. Por lo tanto, la PP se acercará a $100 \%$, en la medida que el IP combinado sea más alto.

\section{¿Es necesario que se estudie a la madre?}

Aunque se podría asumir que en un estudio de paternidad no es necesario incluir a la madre, la posibilidad de identificar con certeza el alelo here- 
Tabla 3. Perfiles genéticos, IP combinado y PP de un caso en que se asigna paternidad y se incluye a la madre

\begin{tabular}{|lcccccccc|}
\hline Marcador & Alelos de la madre & \multicolumn{2}{c}{ Alelos del hijo/a } & \multicolumn{2}{c|}{ Alelos del p-Padre } & IP \\
\hline D3S1358 & 15 & 15 & 15 & 16 & 15 & 16 & 2,43 \\
WWA & 16 & 17 & 17 & 18 & 15 & 18 & 3,27 \\
\hline FGA & 23 & 25 & 23 & 23 & 21 & 23 & 3,12 \\
\hline D8S1179 & 11 & 13 & 11 & 15 & 13 & 15 & 4,26 \\
\hline D21S11 & 29 & 31 & 29 & 31 & 29 & 30 & 2,10 \\
\hline D18S51 & 17 & 18 & 17 & 18 & 13 & 17 & 2,22 \\
\hline D5S818 & 11 & 13 & 12 & 13 & 11 & 12 & 2,13 \\
\hline D13S317 & 8 & 11 & 8 & 12 & 12 & 12 & 4,55 \\
\hline D7S820 & 10 & 11 & 11 & 12 & 12 & 12 & 5,92 \\
\hline D16S539 & 11 & 14 & 11 & 14 & 11 & 13 & 1,57 \\
\hline TH01 & 9 & 9,3 & 6 & 9,3 & 6 & 7 & 1,62 \\
\hline TPOX & 8 & 8 & 8 & 8 & 8 & 11 & 1,06 \\
\hline CSF1PO & 11 & 12 & 11 & 12 & 12 & 12 & 3,07 \\
\hline D2S1338 & 19 & 19 & 19 & 20 & 20 & 22 & 3,14 \\
\hline D19S433 & 13 & 15,2 & 15 & 15,2 & 15 & 15 & 5,50 \\
\hline IP combinado & & & & 4.018 .051 & $99,9999 \%$ & & \\
\hline Probabilidad de Paternidad & & & & & & \\
\hline
\end{tabular}

dado de la madre permite definir el alelo paterno obligado y alcanzar IP más altos y probabilidades de paternidad también más altas y, por lo tanto, más seguras. Cuando no se estudia a la madre, se deben utilizar fórmulas que, comparadas con la que se aplica para casos con la madre, disminuyen el IP combinado y la $\mathrm{PP}^{8}$. En el ejemplo de la Tabla 3 , el estudio incluye a la madre, con lo que se obtiene un IP combinado de 4.018.051 y una PP de $99,9999 \%$. En cambio, si en el mismo caso la madre no se estudia, el IP disminuye a 939 (4.000 veces menor) y la probabilidad de paternidad a $99,89 \%$. De acuerdo a la normativa emitida por el Servicio Médico Legal el año 2000, la paternidad biológica se considera acreditada cuando la probabilidad de paternidad es mayor al $99,9 \%{ }^{7}$.

En casos de exclusión de paternidad, el incluir a la madre también hace más seguro el estudio de paternidad, porque permite tomar en cuenta sólo el alelo paterno obligado para definir si hay exclusión de paternidad. En cambio, si sólo se estudian supuestos padre e hijo/a, los dos alelos del hijo/a deberán estar ausentes en el supuesto padre para que se pueda excluir paternidad; por lo tanto, se encontrarán menos STR que permitan excluir la paternidad que en un caso en que se estudie también a la madre. En el ejemplo de la Tabla 2, el número de STR que excluye paternidad aumenta de 6 a 9 marcadores, si se incluye a la madre en el estudio. Esto adquiere especial relevancia, si hay parentesco entre dos presuntos padres.

\section{Calidad, aspectos éticos y legales}

La fuerza legal de la prueba de ADN para el estudio de paternidad está ampliamente aceptada en Chile, lo que se refleja en las modificaciones introducidas ya en el año 2000 a nuestra ley de filiación ${ }^{9}$.

En nuestro país es posible acceder a realizarse el estudio de paternidad en forma privada o por solicitud de un juez. Para los estudios realizados en forma privada existen aun algunas interrogantes, por ejemplo, respecto a que en algunos laboratorios sea posible que un hombre, aún no siendo el 
padre legal de un niño/a menor de edad, pueda acceder a un estudio de paternidad sin el conocimiento y consentimiento de la madre o tutor legal.

Los estudios solicitados a través de un tribunal se realizan en el Servicio Médico Legal y también en laboratorios privados, si los involucrados asumen los costos del examen. Sin embargo, para que estos resultados tengan validez legal, los laboratorios deben estar habilitados por el Servicio Médico Legal de Chile y cumplir con las exigencias de la normativa emitida por este Servicio ${ }^{7}$.

Dada la implicancia que tiene un estudio de paternidad en la vida de un ser humano, es importante que los laboratorios donde se realicen estudios de paternidad, tengan éstos validez legal o no, estén inmersos en un sistema de aseguramiento de la calidad, incluyendo aspectos como confidencialidad, cadena de custodia, control de calidad, entre otros.

\section{Cierre del caso}

En el caso presentado, la probabilidad de paternidad es de $99,89 \%$, lo que corresponde a una paternidad biológica altamente probable, pero no alcanza el mínimo aceptado para paternidad biológica acreditada (>99,9\%), de acuerdo a la normativa vigente en Chile y a lo aceptado por los tribunales de familia de nuestro país. Al no estudiar a la madre, estando ella disponible, se le restó fuerza estadística al estudio, por lo que se debería recomendar que se le incluyera para así alcanzar una probabilidad $>99,9 \%$. Otro antecedente a considerar es que los estudios de paternidad sin la madre (dúos) tienen un costo similar a los que la incluyen (tríos). Si en cualquier caso existen dudas sobre la probabilidad de paternidad, es posible estudiar marcadores genéticos adicionales.

\section{Referencias}

1. Albrecht K, Schultheiss D. Proof of paternity: historical reflections on an andrological-forensic challenge. Andrologia 2004; 36: 31-7.

2. Butler JM. Forensic DNA Typing: Biology, Technology, and Genetics of STR Markers, (2005) 2nd edition. NewYork: Elsevier.

3. Jorquera GH, Acuña PM, Cifuentes LL. Estudios de parentesco mediante marcadores del ADN: Experiencia en resolución de casos en los últimos años. Rev Med Chile 2008; 136: 193-200.

4. Kristt D, Stein J, Yaniv I, Klein T. Assessing quantitative chimerism longitudinally: technical considerations, clinical applications and routine feasibility. Bone Marrow Transplant 2007; 39: 255-68.

5. STR data base. Disponible en http://www.cstl.nist.gov/ strbase (Consultado el 22 marzo de 2011).

6. Butler JM. Short tandem repeat typing technologies used in human identity testing. Biotechniques 2007; 43: Sii-Sv.

7. Instrucciones y normativa técnica sobre pruebas biológicas para la determinación de la paternidad y/o maternidad. Diario Oficial/Normas Generales/Año 2000/DO 08/01/2000 RES 1.450 2000. Servicio Médico Legal.

8. Wenk RE, Houtz T, Chiafari FA. Maternal typing and test sufficiency in parentage analyses. Transfusion 2006; 46:199-203.

9. Ley de Filiación 19.585 Diario Oficial de 26 de octubre 1998. Resolución Exenta № 1.450 de 29 diciembre 1999, Diario Oficial 8 enero 2000. 